\title{
The Growth of Transition Metals on H-Passivated Si(111) Substrates**
}

\author{
By Jan O. Hauch, Mikhail Fonine, Ulrich May, Raffaella Calarco, Harish Kittur, Jin M. Choi, \\ Ulrich Rüdiger,* and Gernot Güntherodt
}

The growth of Co and Ag layers on wet-processed $\mathrm{H}$-passivated $\mathrm{Si}(111)$ substrates by molecular beam epitaxy (MBE) has been studied using high resolution scanning tunneling microscopy (STM) with regard to possible applications of the layers in magnetoelectronic devices. Roughness and intermixing at interfaces as functions of deposition temperature and layer thickness are key parameters for the performance of such devices. The initial growth of $\mathrm{Co}$ and $\mathrm{Ag}$ and the influence of $\mathrm{Ag}$ atoms on the $\mathrm{Si}(111)$ surface reconstructions provide insight into adatom-substrate interactions.

\section{Introduction}

To optimize the structure-property relationship of layered systems exhibiting giant magnetoresistance (GMR) ${ }^{[1-3]}$ or tunneling magnetoresistance (TMR) ${ }^{[4-6]}$ there is a call for textured or epitaxial ferromagnetic layers deposited on semiconductor substrates. For high-performance GMR and TMR devices the interface properties (roughness, electronic interface states, spin polarization, phase purity, etc.) of the ferromagnetic/non-ferromagnetic metal layers (GMR) and ferromagnetic metal/insulator hybrid structures (TMR) play a crucial role.

Currently, the "state-of-the-art" theoretical models for GMR $^{[7-9]}$ and TMR systems ${ }^{[10,11]}$ are mainly developing calculations for epitaxial layers. Therefore the experimental investigation of epitaxial model systems becomes indispensable.

In order to grow high-quality (textured or epitaxial) Co thin films on oxide-free Si substrates the possible advantages of $\mathrm{H}$ passivated $\mathrm{Si}(111)$ substrates have been explored. The latter can be prepared by "ex situ" wet-chemical processes and introduced easily into ultra high vacuum (UHV). The most reliable and reproducible results have been achieved on H-passivated Si(111) surfaces, ${ }^{[12]}$ although this crystal orientation may not yield continuous epitaxial growth of face-centered cubic (fcc) metal layers. ${ }^{[13,14]}$ In order to avoid silicide formation at the $\mathrm{Co}-\mathrm{Si}$ interface, the influence of $\mathrm{Ag}$ buffer layers has been investigated.

The influence of $\mathrm{Ag}$ deposition at elevated temperatures ("hot" $\mathrm{Ag}$ deposition at $T=550{ }^{\circ} \mathrm{C}$ ) on the formation of $\operatorname{Si}(111)-(2 n+1) \times(2 n+1)$ reconstructions will be discussed and compared with the results of the reconstruction formation on a $\mathrm{H}$-Si(111) surface after H-desorption by thermal annealing. The influence of Ag deposited at a lower temperature ("cold"

[*] Dr. U. Rüdiger, J. O. Hauch, M. Fonine, Dr. U. May, Dr. R. Calarco, H. Kittur, Dr. J. M. Choi, Prof. G. Güntherodt

II. Physikalisches Institut, RWTH Aachen

D-52056 Aachen (Germany)

E-mail: ruediger@physik.rwth-aachen.de

[**] This work was supported by Innovationsprogramm Forschung Forschungsverbund "Nanowissenschaften" of Nordrhein-Westfalen. J. M. Choi thanks the DAAD, Germany, and KOSEF, Korea, for financial support.
Ag deposition at $T=250{ }^{\circ} \mathrm{C}$ ) on the "wet prepared" $\mathrm{H}-\mathrm{Si}(111)$ surface has also been investigated and compared with the hot Ag deposition at $550^{\circ} \mathrm{C}$ on $\mathrm{H}-\mathrm{Si}(111)$.

In this paper, growth modes of $\mathrm{Co}$ and $\mathrm{Ag}$ on $\mathrm{H}$-passivated $\mathrm{Si}(111)$ substrates will be presented and discussed with regard to possible applications for magnetoelectronic devices. ${ }^{[15,16]}$

\section{Results and Discussion}

After introduction of a wet-chemically prepared monohydride $\mathrm{Si}(111)-(1 \times 1)$ substrate into UHV, the quality and cleanliness of the surface were checked by scanning tunneling microscopy (STM) and Auger electron spectroscopy (AES). The Auger spectra of wet-chemically etched H-Si(111) substrates show a typical Si Auger LMM transition peak at an energy of $92 \mathrm{eV}$. The Auger intensities of carbonates and oxygen, which are the main contaminants, are negligible. From the Auger spectra one can conclude that the samples are clean on a macroscopic scale.

Figure 1 shows a STM image of a H-passivated $\mathrm{Si}(111)$ surface of $1000 \mathrm{~nm} \times 1000 \mathrm{~nm}$. The triangular shape of the $\mathrm{H}$ $\mathrm{Si}(111)$ surface is due to the anisotropic etching rates along the

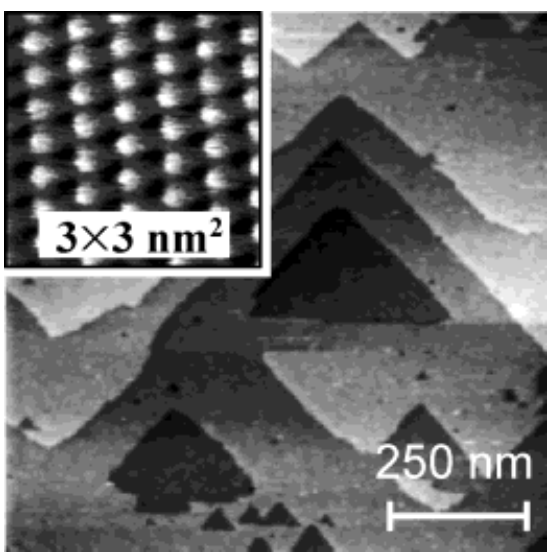

Fig. 1. STM image of large-area, triangular terraces of a wet-chemically processed H-terminated $\mathrm{Si}(111)$ surface. The inset shows that the monohydride termination leads to a $\mathrm{H}-\mathrm{Si}(111)-(1 \times 1)$ surface. 
[111]- and [100]-microfacet directions. ${ }^{[17]}$ The step height was determined to be $3.1 \AA$, which corresponds to the height of one $\mathrm{Si}$ double layer, which is typical for the $\mathrm{Si}(111)$ surface. The atomically flat triangular terraces of the wet-chemically prepared $\mathrm{H}-\mathrm{Si}(111)-(1 \times 1)$ surface extend over hundreds of nanometers with no evidence of significant contamination. The inset in Figure 1 shows the atomically resolved H-passivated Si(111) surface with a lattice constant of $3.89 \AA$.

The outstanding surface properties and the "easy-to-handle" $\mathrm{H}$-passivation procedure of $\mathrm{Si}(111)$ substrate surfaces make them promising templates for investigating growth and texture of ferromagnetic transition metals for application in magnetoelectronic devices on the basis of established Si-technology.

\subsection{Co-Growth at Room Temperature}

Figure 2a shows a STM image of a H-Si(111) surface with nominally 0.5 monolayers (ML) of Co deposited at room temperature (RT). The STM image reveals an island-like growth on
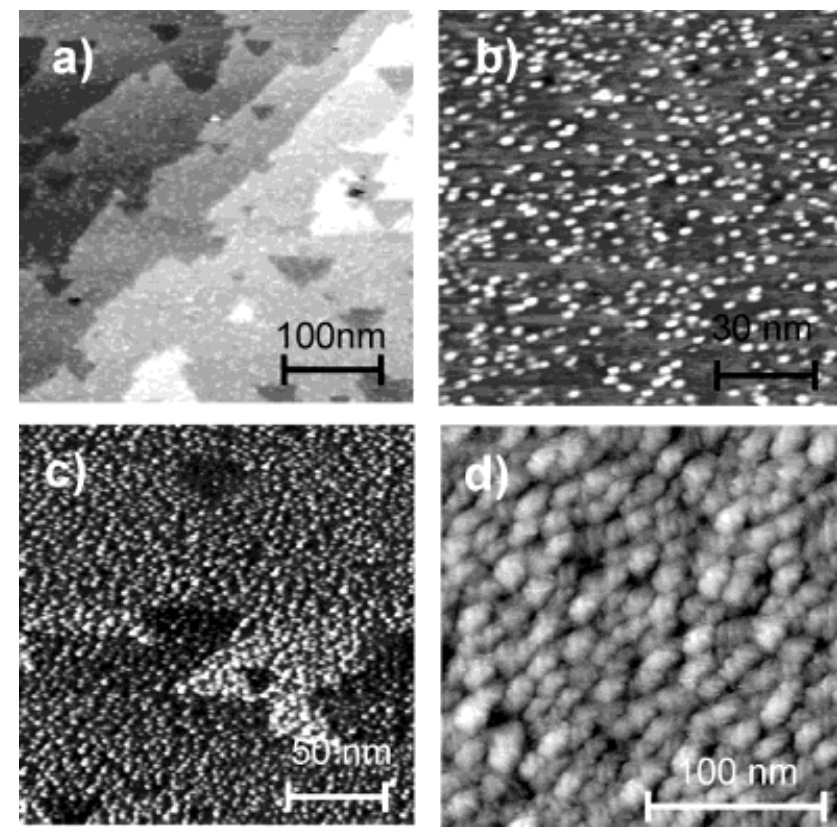

Fig. 2. H-passivated $\mathrm{Si}(111)$ surface covered at RT by nominally a) $0.5 \mathrm{ML} \mathrm{Co}$, b) $1 \mathrm{ML} \mathrm{Co,} \mathrm{c)} 7 \mathrm{ML} \mathrm{Co}$, and d) $100 \mathrm{ML}$ Co.

the triangular, atomically flat $\mathrm{H}-\mathrm{Si}(111)$ substrate surface. The islands have an average diameter of 30-50 $\AA$ and an average height of $5.5 \AA$. The coverage of the surface was determined to be approximately $12 \%$. The STM image clearly identifies a Vollmer-Weber growth mode for the Co on the H-Si(111) surface at RT. As the nominal thickness is increased from $1 \mathrm{ML}$ (Fig. 2b) to $7 \mathrm{ML}$ (Fig. 2c), the growth and evolution of the Co cluster size and density as well as the roughness can be shown. Figure $2 \mathrm{~b}$ shows a $120 \mathrm{~nm} \times 120 \mathrm{~nm}$ region of the $\mathrm{H}-\mathrm{Si}(111)$ surface covered by nominally 1 ML Co at RT. The diameter of the Co clusters is around $40 \AA$ and the peak-to-peak roughness of $5.5 \AA$ is in the same range as in Figure 2a. In Figure $2 \mathrm{c}$ (nominally $7 \mathrm{ML}$ Co, grown at RT, scan size $200 \mathrm{~nm} \times 200 \mathrm{~nm}$ ) the clusters start to merge, causing an increase of the peak-to-peak roughness to $6.5 \AA$. The observed strong cluster formation of the deposited Co clusters is probably due to the weak Co-substrate interaction across the hydrogen atoms of the H-passivated $\mathrm{Si}(111)$ surface and the large lattice mismatch between the $\mathrm{Co}(0001)$ surface with a lattice constant of $2.54 \AA$ and the Si(111) surface with $3.89 \AA$. The Co-Co binding energy of approximately $1 \mathrm{eV}^{[18]}$ is much larger than the van der Waalslike $\mathrm{H}-\mathrm{Co}$ interaction. The large surface diffusion of Co atoms at RT supports the tendency of Co cluster formation. ${ }^{[19]}$ $\mathrm{H}$-desorption due to the presence of Co atoms on top of the $\mathrm{H}$-passivation layer was not observed with STM imaging. In the case of H-desorption, Si dangling bonds would lead to a strong contrast in the STM images of the H-Si(111) surface.

Finally, Figure 2d shows a STM image of a $200 \AA$ thick Co layer on $\mathrm{H}-\mathrm{Si}(111)$ deposited at RT. The average lateral size of the Co clusters is about $300 \AA \times 180 \AA$. The peak-to-peak roughness was determined to be $18 \AA$. The STM image clearly exhibits an anisotropic in-plane growth of the Co clusters, but the growth anisotropy of the Co clusters has not yet been successfully correlated with the sixfold in-plane symmetry of the $\mathrm{H}-\mathrm{Si}(111)$ substrate surface. The resulting roughness of a $200 \AA$ thick Co layer is comparable with the typical thickness of the insulator barrier (10-20 ̊) in magnetic tunnel junctions. This may cause problems in depositing pinhole-free, homogeneous insulator layers on top of the bottom electrode of such tunnel junctions using this type of H-passivated $\mathrm{Si}(111)$ substrates.

The Co growth at RT was also examined by reflection high energy electron diffraction (RHEED). In Figure 3a a RHEED
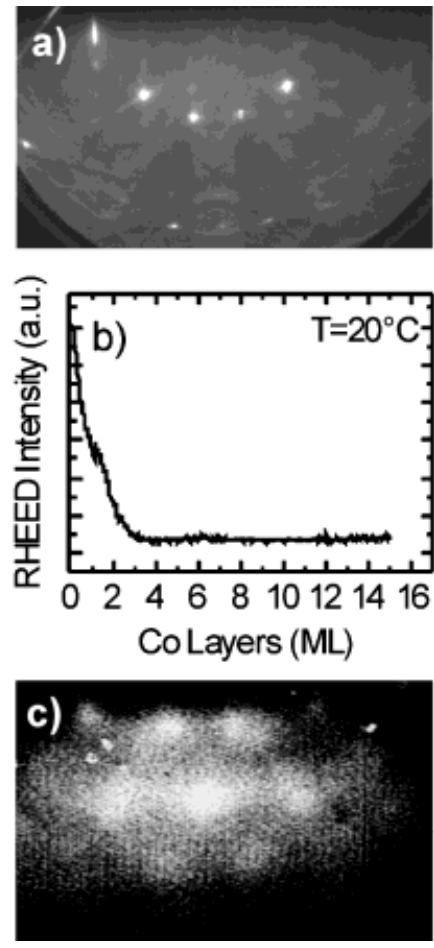

Fig. 3. a) The RHEED pattern with reflection spots on Laue circles demonstrates a flat and well-ordered H-Si(111) surface. b) The RHEED intensity of the 10 reflection as a function of Co coverage shows an exponential decrease, which is due to the 3D growth of Co islands. c) The sixfold symmetry of the 3D RHEED pattern of a nominally 15 ML thick Co layer indicates a slight $c$-axis orientation of the Co clusters. 
pattern of a pure $\mathrm{H}-\mathrm{Si}(111)$ substrate is shown. The position of reflection spots on Laue circles is an indication of a flat and well-ordered H-Si(111) surface, as was also seen in the STM investigation of the substrates. Figure $3 b$ shows the RHEED intensity of the 10 spot during the growth of nominally $15 \mathrm{ML}$ Co. A rapid drop of the RHEED intensity within the first $3 \mathrm{ML}$ is due to three-dimensional (3D) island growth of Co on H-passivated $\mathrm{Si}(111)$ substrates, corresponding to the accumulated roughness with increasing Co thickness, as was also observed in the STM studies. After deposition of nominally $15 \mathrm{ML} \mathrm{Co,} \mathrm{a}$ 3D RHEED pattern appears, indicating a weak texture of the Co clusters (see Fig. 3c). The large lattice mismatch between the $\mathrm{Co}(0001)$ surface with a lattice constant of $2.54 \AA$ and the Si(111) surface with $3.89 \AA$ makes a simple in-plane relationship of the two types of surface orientations unlikely.

\subsection{Co-Growth at Elevated Temperature}

In order to reduce the roughness of a thick Co film, Co was deposited at an increased substrate temperature. H-passivated Si substrates were used in an attempt to avoid the well-known cobalt silicide, $\mathrm{CoSi}_{x}$, formation at higher temperatures. ${ }^{[20]}$ Figure 4 shows a STM image of nominally $1 \mathrm{ML}$ Co on H-passivated $\mathrm{Si}(111)$ deposited at a substrate temperature of $100^{\circ} \mathrm{C}$ (scan size $156 \mathrm{~nm} \times 156 \mathrm{~nm}$ ). The cluster-like growth mode is

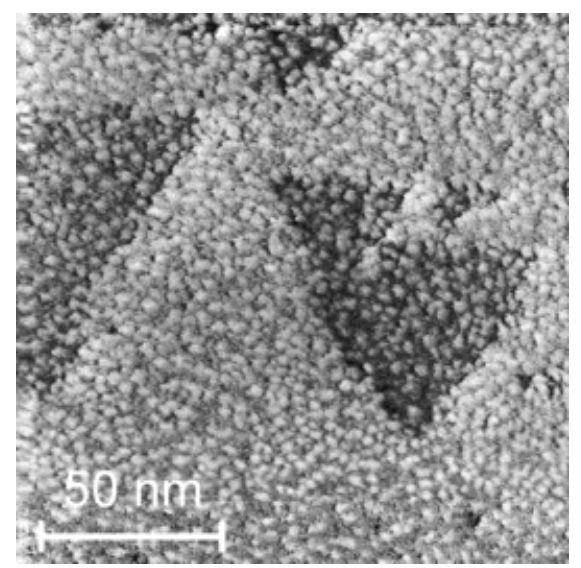

Fig. 4. STM image of nominally $1 \mathrm{ML}$ Co on $\mathrm{H}-\mathrm{Si}(111)$ deposited at $T=100^{\circ} \mathrm{C}$. Beside the Co cluster formation a significant change of the $\mathrm{H}-\mathrm{Si}(111)$ surface topography appears due to $\mathrm{CoSi}_{x}$ formation.

similar to the growth at RT. The clusters exhibit a height and diameter distribution comparable to that for the RT growth (see Fig. 2b). Beside the Co cluster formation, the topography (roughness) of the underlying H-passivated $\mathrm{Si}(111)$ substrate surface has changed dramatically. It was concluded that during the high temperature growth of $\mathrm{Co}$ the $\mathrm{H}-\mathrm{Si}$ bonds break and $\mathrm{CoSi}_{x}$ formation takes place. AES investigation of a $200 \AA$ thick Co film also showed the presence of Si atoms within the probed depth, indicating $\mathrm{CoSi}_{x}$ formation during the growth of the Co film at a temperature of $100^{\circ} \mathrm{C}$. The change of the magnetic bulk and surface properties of Co electrodes due to $\operatorname{CoSi}_{x}$ formation is detrimental for the application of such a growth process in fabricating magnetoelectronic devices such as mag- netic tunnel junctions (MTJs). For TMR and GMR multilayers the interface properties (electronic states, spin polarization, roughness) at the ferromagnet-insulator interface are crucial parameters for the performance of the devices. In this case an uncontrolled formation of Co silicides $\left(\mathrm{CoSi}_{x}\right)$ at the cobalt-insulator interface has to be avoided.

\subsection{Co-Growth with Ag Buffer Layer}

To avoid $\mathrm{CoSi}_{x}$ formation and to lower the film roughness, metallic buffer layers can be deposited on the H-passivated $\mathrm{Si}(111)$ substrate. For this propose Ag was chosen as the buffer layer because the growth of $\mathrm{Ag}$ on $\mathrm{Si}$ has already been studied $^{[21,22]}$ and the immiscibility of $\mathrm{Ag}$ with both $\mathrm{Si}^{[23]}$ and $\mathrm{Co}^{[24]}$ has been proved.

In Figure 5a a $200 \AA$ Ag buffer layer grown on H-Si(111) at RT is shown. Atomically flat terraces with a local sixfold
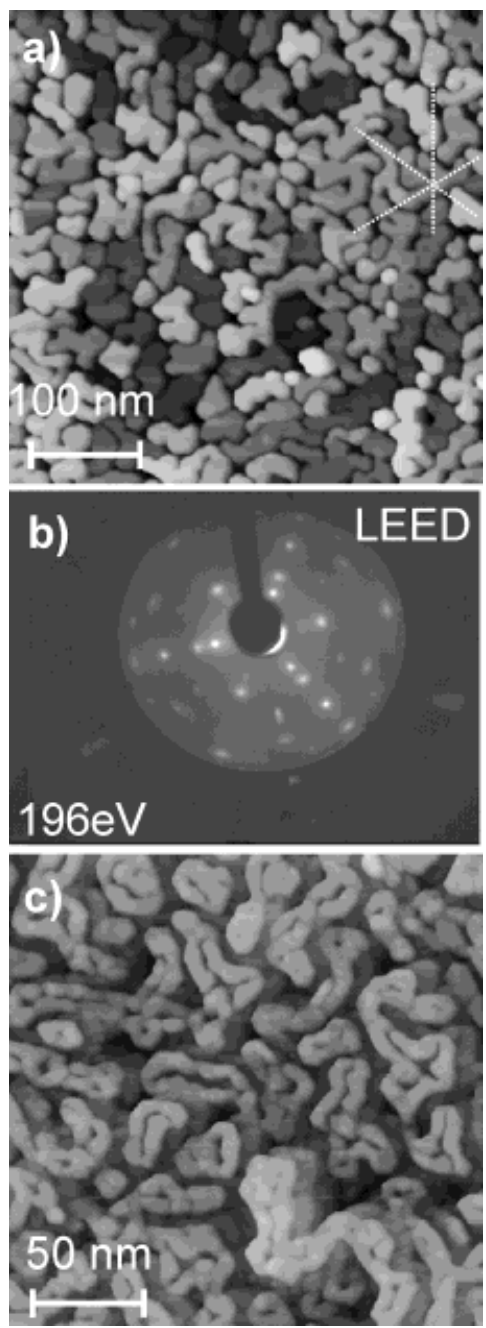

Fig. 5. a) STM image of a maze-like island structure with a sixfold in-plane symmetry (see dotted guidelines) of a nominally $200 \AA$ thick Ag layer on a H-Si(111) substrate. b) The corresponding LEED pattern at an electron energy of $196 \mathrm{eV}$ with a clear sixfold symmetry due to (111) orientation of the Ag layer. c) STM image of a nominally $200 \AA$ thick Co layer on a (111)-oriented thick Ag buffer layer with a maze-like island structure. The Co cluster formation appears along the edges of the Ag islands. 
in-plane symmetry (see dotted guidelines) can be seen due to a (111) orientation of the Ag layer. The low energy electron diffraction (LEED) pattern in Figure 5b of the Ag buffer layer shows also a sixfold symmetry, which confirms the (111) orientation of the Ag film on the H-passivated Si(111) surface. The lattice mismatch of a $\mathrm{Ag}(111)$ layer with a lattice parameter of $3.53 \AA$ on the $\mathrm{Si}(111)$ surface with $3.89 \AA$ is much smaller than in the case of $\mathrm{Co}(111)$ on $\mathrm{Si}(111)$. Nevertheless, the observed flat terraces are separated by deep trenches. The depth of the trenches could not be determined by STM, but in the corresponding Auger spectrum (not shown here) of the Ag film a clear Si Auger LMM transition was observed at an electron energy of $92 \mathrm{eV}$. As there is no intermixing between $\mathrm{Si}$ and $\mathrm{Ag}$ at RT, these trenches seem to go through the whole Ag layer.

Figure 5c shows the STM image of a $200 \AA$ thick Co film on a $200 \AA$ thick Ag buffer layer on a H-Si(111) substrate grown at RT. The growth of Co on top of the $200 \AA$ thick Ag buffer layer leads to elongated Co clusters, which extend along the edges of the Ag islands. Here also, the deposition of a Ag buffer layer does not give the smoother and more homogeneous Co films necessary for ferromagnetic electrodes in magnetoelectronic devices.

\subsection{Influence of Ag Deposition at Elevated Temperatures on the H-Passivated Si(111) Surface}

The wet-chemically prepared $\mathrm{H}-\mathrm{Si}(111)-(1 \times 1)$ substrate was heated in UHV up to $550^{\circ} \mathrm{C}$ and annealed for $40 \mathrm{~min}$. Annealing at this temperature should lead to a complete desorption of the hydrogen from the $\mathrm{H}-\mathrm{Si}(111)$ surface. Then $1 \mathrm{ML}$ of $\mathrm{Ag}$ was deposited at $550{ }^{\circ} \mathrm{C}$ (hereafter this procedure will be called hot deposition of $\mathrm{Ag}$ ). Once the $\mathrm{Ag}$ deposition had been finished, the temperature of the sample was maintained at $550{ }^{\circ} \mathrm{C}$ for $30 \mathrm{~min}$. Then the sample was cooled down to RT. Figure 6 shows a STM image of the $\mathrm{Si}(111)$ surface resulting after hydrogen desorption followed by the hot deposition of Ag. Different dimer-adatom-stacking fault (DAS) structures with the periodicity of $(2 n+1) \times(2 n+1)^{[25]}$ can be found on the $\operatorname{Si}(111)$

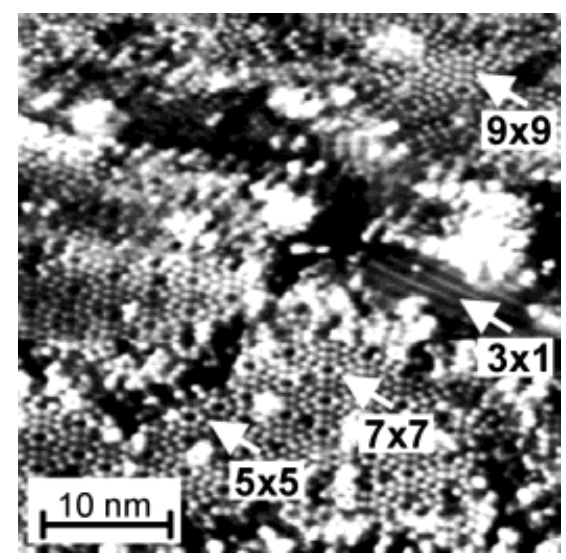

Fig. 6. STM image of a Si(111) surface after hydrogen desorption from a wetchemically prepared $\mathrm{H}-\mathrm{Si}(111)-(1 \times 1)$ sample at $550^{\circ} \mathrm{C}$ followed by the deposition of $1 \mathrm{ML} \mathrm{Ag}$ at the same temperature. The observable $\mathrm{Ag} / \mathrm{Si}(111)-(3 \times 1)$ and $\mathrm{Si}(111)-((2 n+1) \times(2 n+1))$ reconstructions are marked by arrows. surface, i.e., $\operatorname{Si}(111) 3 \times 3,5 \times 5,7 \times 7$, and $9 \times 9$ reconstructions. Magnifications of these reconstructions are shown in Figures $7 \mathrm{a}-\mathrm{d}$. A fragment of the Ag-Si(111)- $(3 \times 1)$ reconstruction $^{[26]}$ is also present on the surface (see Fig. 6). Such frag-
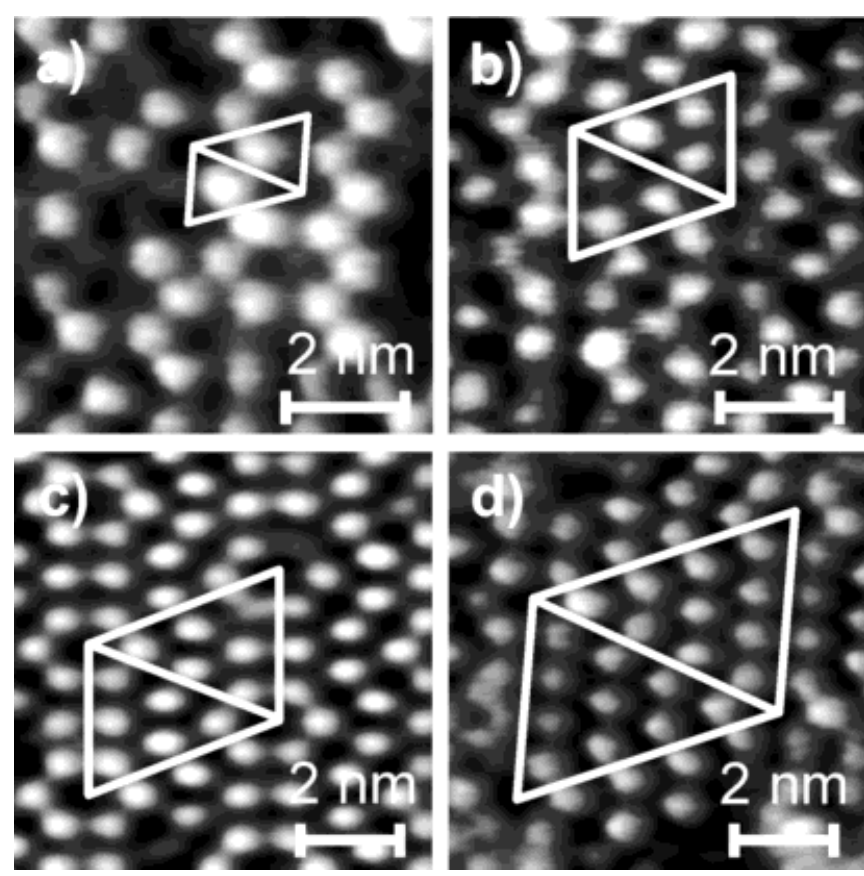

Fig. 7. Magnifications of observed reconstructions in Figure 6 after deposition of $1 \mathrm{ML} \mathrm{Ag}$ at $550^{\circ} \mathrm{C}$ on the $\mathrm{Si}(111)$ surface (hot deposition of $\mathrm{Ag}$ ): a) $\mathrm{Si}(111)$ $(3 \times 3)$, b) $\operatorname{Si}(111)-(5 \times 5)$, c) $\operatorname{Si}(111)-(7 \times 7)$, and d) $\operatorname{Si}(111)-(9 \times 9)$.

ments were found over the whole surface investigated. No areas with a $\mathrm{Ag}$-Si(111) $-(\sqrt{3} \times \sqrt{ } 3)$ reconstruction were observed on the sample surface. This may be due to the low coverage, i.e., $1 \mathrm{ML}$ of $\mathrm{Ag}$. For another sample with $3 \mathrm{ML}$ of $\mathrm{Ag}$ deposited under the same conditions, large areas of a $\mathrm{Ag}-\mathrm{Si}(111)$ $(\sqrt{3} \times \sqrt{3})$ reconstruction were found.

It is known that the $\mathrm{Ag}-\mathrm{Si}(111)-(\sqrt{3} \times \sqrt{3})$ phase can be formed by $\mathrm{Ag}$ deposition on the $7 \times 7$ surface at temperatures in the range of $220-550{ }^{\circ} \mathrm{C} .{ }^{[27,28]}$ Around $550{ }^{\circ} \mathrm{C} \mathrm{Ag}$ starts to desorb from the Ag-Si(111)- $(\sqrt{3} \times \sqrt{3})$ surface. As Ag desorbs from the surface, $3 \times 1$ and $6 \times 1$ phases also generally appear, besides the $\sqrt{3} \times \sqrt{3}$ structure, depending on the amount of Ag removed from the surface. ${ }^{[26]}$ This means that the observed $3 \times 1$ reconstruction (Fig. 6) gives clear evidence of the existence of $\mathrm{Ag}$ on the sample surface. During the formation of $3 \times 1$ reconstruction areas the $\mathrm{Si}$ atoms are displaced by $\mathrm{Ag}$ and this may also be the source of the Si atoms.

The formation of the $\mathrm{Ag}-\mathrm{Si}(111)-(\sqrt{3} \times \sqrt{3})$ phase is known to involve a substantial redistribution within the top $\mathrm{Si}(111)$ substrate layer (i.e., surface Si mass transport). ${ }^{[29]}$ Silicon transport as evidenced by $\mathrm{Ag}-\mathrm{Si}$ interaction ${ }^{[30-32]}$ can be attributed to the enhancement of the surface migration of Si atoms under Ag irradiation at elevated temperatures. During the $\mathrm{Ag}$ evaporation onto the $\mathrm{Si}(111)$ substrate at $550{ }^{\circ} \mathrm{C}, \mathrm{Si}$ atoms of the top substrate layers may be more mobile than without the Ag atom flux.

The disordered high-temperature " $1 \times 1$ " structure on the $\mathrm{Si}(111)$ surface is considered to be energetically closer to the 
$\sqrt{3} \times \sqrt{3}$ reconstruction, because this disordered structure contains Si adatoms on the surface, as suggested by Yang and Williams. ${ }^{[33]}$ According to theoretical total energy calculations, the difference of total energy between ideal $1 \times 1$ and $7 \times 7$ structures $^{[34]}$ and between ideal $1 \times 1$ and $\sqrt{3} \times \sqrt{3}$ structures $^{[35]}$ are $0.4 \mathrm{eV}$ and $0.3 \mathrm{eV}$ per $1 \times 1$ unit cell, respectively. The Ag on the $\mathrm{Si}(111)-(1 \times 1)$ surface may induce $\mathrm{Si}$ adatom formation, which supports the formation of the DAS structures.

After the sample with a nominal coverage of $1 \mathrm{ML} \mathrm{Ag}$ on the surface was annealed at a temperature of $600^{\circ} \mathrm{C}, \mathrm{Ag}$ was desorbed from the surface. All the superstructures observed on this sample previously were converted into the almost perfect $7 \times 7$ reconstruction. The images obtained in pseudo-constantheight STM mode (high scan speed, feedback loop set to minimum) with a sample bias of $-2.51 \mathrm{~V}$ showed a difference in the surface electronic states between the stacking faulted (SF) regions and stacking unfaulted (UF) regions in the $7 \times 7$ unit cell (not shown here). This proves that the images show the real $7 \times 7$ reconstruction.

The annealing temperature of $600{ }^{\circ} \mathrm{C}$ is far below the temperature at which the $(1 \times 1) \rightarrow(7 \times 7)$ transformation occurs $\left(860^{\circ} \mathrm{C}\right)$ and is also below the temperature of the $7 \times 7$ structure formation observed for thermal hydrogen desorption and annealing experiments at $700{ }^{\circ} \mathrm{C}$ on the wet processed $\mathrm{H}-\mathrm{Si}(111)$ surface. ${ }^{[36]}$ The possibility of $7 \times 7$ structure formation at temperatures lower than $700^{\circ} \mathrm{C}$ was also shown by RHEED in the thermal H-desorption experiments on the wet-processed H-Si(111) surface. ${ }^{[37]}$

After deposition of nominally $3 \mathrm{ML} \mathrm{Ag}$ onto a wet-processed $\mathrm{H}-\mathrm{Si}(111)$ surface at a temperature of only $T=250^{\circ} \mathrm{C}$ (cold deposition of $\mathrm{Ag}$ ), which is below the hydrogen desorption temperature (around $450{ }^{\circ} \mathrm{C}$ ), Ag was thermally desorbed from the $\mathrm{Ag} / \mathrm{H}-\mathrm{Si}(111)$ surface. Here the $\mathrm{Ag}$ desorption temperature was chosen to be around $700{ }^{\circ} \mathrm{C}$ to ensure that the $\mathrm{Ag}$ is desorbed from the $\mathrm{Ag}-\mathrm{Si}(111)-(\sqrt{3} \times \sqrt{3})$ surface. At a lower temperature of approximately $450{ }^{\circ} \mathrm{C}$ the hydrogen atoms first desorb from the $\mathrm{Ag} / \mathrm{H}-\mathrm{Si}(111)$ surface. Then, at around $550{ }^{\circ} \mathrm{C}$, $\mathrm{Ag}$ forms the $\mathrm{Ag}-\mathrm{Si}(111)-(\sqrt{3} \times \sqrt{3})$ reconstruction as shown in Figure $8 .^{[38]}$ After the desorption of the Ag from the Ag-

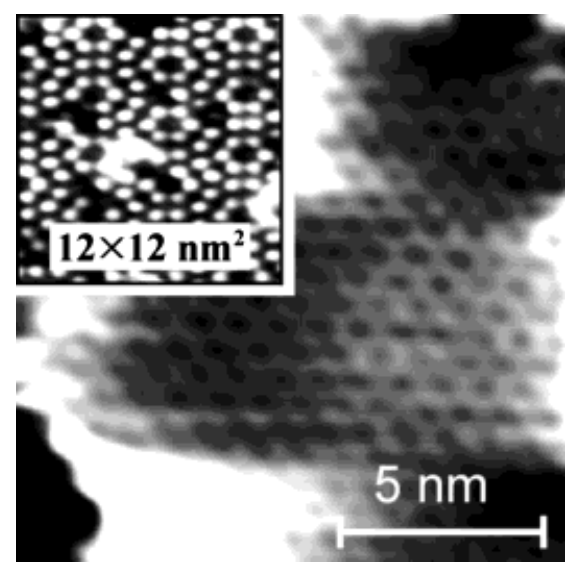

Fig. 8. STM image of the $\operatorname{Si}(111)$ surface $(\operatorname{Ag} / \operatorname{Si}(111)-(\sqrt{3} \times \sqrt{ } 3))$ after the deposition of $3 \mathrm{ML} \mathrm{Ag}$ at $T=250^{\circ} \mathrm{C}$ (cold deposition of $\mathrm{Ag}$ ) and an annealing step a $550^{\circ} \mathrm{C}$ for $40 \mathrm{~min}$. The inset shows the same surface after additional annealing at $T=700^{\circ} \mathrm{C}$ for $120 \mathrm{~min}$; a clear $\mathrm{Si}(111) 7 \times 7$ reconstruction can be seen.
$\operatorname{Si}(111)-(\sqrt{3} \times \sqrt{3})$ surface at a temperature between $600^{\circ} \mathrm{C}$ and $700{ }^{\circ} \mathrm{C}$ a transition of the surface to the $7 \times 7$ reconstruction takes place. As a result of this annealing experiment a clear $7 \times 7$ reconstruction could be seen (inset in Fig. 8). The DAS structure was confirmed by resolving SF and UF regions of the $7 \times 7$ unit cell in the pseudo-constant-current mode with the same sample bias, $-2.51 \mathrm{~V}$. No evidence of other DAS structures such as $3 \times 3,5 \times 5$, and $9 \times 9$ was found. These measurements imply that the hot deposition of $\mathrm{Ag}$ energetically supports the formation of metastable $\operatorname{Si}(111)-(2 n+1) \times(2 n+1)$ reconstructions.

In order to elucidate the role of $\mathrm{Ag}$ in the formation of $\operatorname{Si}(111)-(2 n+1) \times(2 n+1)$ reconstructions, annealing experiments on the wet-processed $\mathrm{H}-\mathrm{Si}(111)$ were performed without $\mathrm{Ag}$ deposition.

Figure 9a shows a STM image of the H-Si(111)-(1×1) surface after annealing at $150{ }^{\circ} \mathrm{C}$ for $10 \mathrm{~min}$. The $1 \times 1$ atomic arrangement of the hydrogen atoms is clearly seen and no significant change of the surface morphology after annealing was observed. The step height of the terraces of $3.1 \AA \pm 0.1 \AA$ is the same as before annealing.
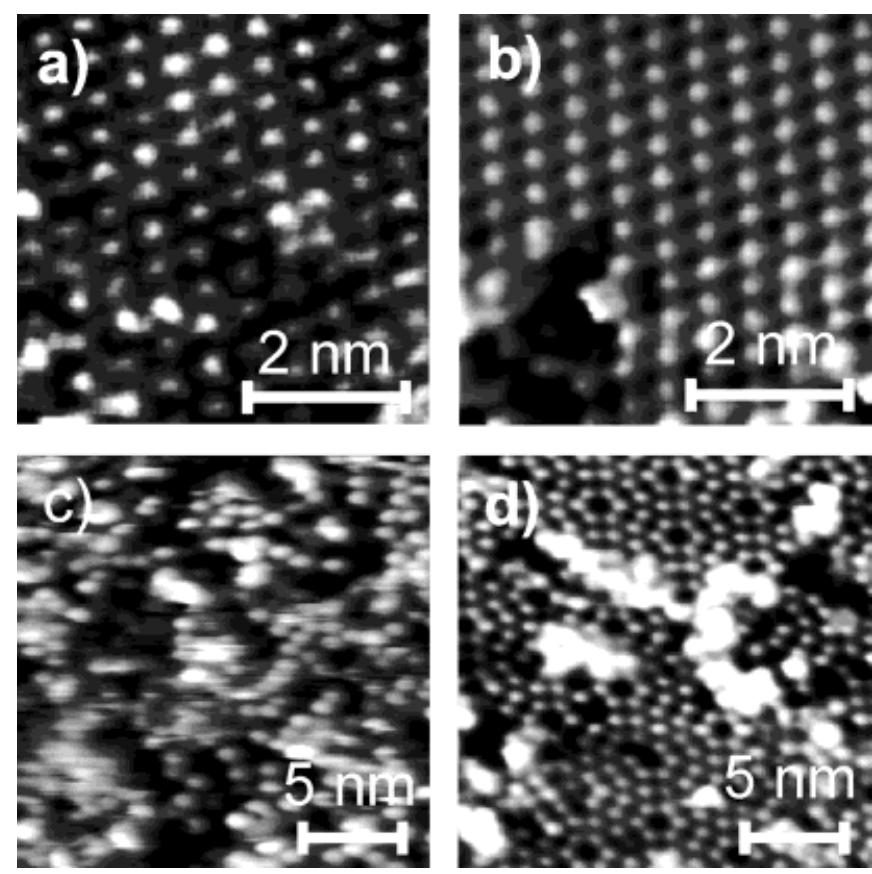

Fig. 9. STM image of a $\mathrm{H}-\mathrm{Si}(111)-(1 \times 1)$ surface a) after annealing at $150^{\circ} \mathrm{C}$ for $10 \mathrm{~min}, \mathrm{~b})$ after annealing at $300^{\circ} \mathrm{C}$ for $15 \mathrm{~min}$, c) distorted after annealing at $450{ }^{\circ} \mathrm{C}$ for $120 \mathrm{~min}$, and d) converted into the $\mathrm{Si}(111) 7 \times 7$ reconstruction after annealing at $650^{\circ} \mathrm{C}$ for $60 \mathrm{~min}$.

After 15 min of additional annealing at $300^{\circ} \mathrm{C}$ of the same sample the terrace shape and also the step height did not change, but a larger number of holes of different shape compared with the initial triangular etch-pits and irregularly shaped clusters appeared on the surface (Fig. 9b). The depth of the pits and the height of the clusters were the same as those on the H-Si(111) surface (around $3 \AA$ ) before annealing. The clusters were resolved atomically and again exhibited a $1 \times 1$ structure (not shown here). It can be seen in Figure $9 \mathrm{~b}$ that the $1 \times 1$ structure is still well arranged without any perturbation 
even if irregularly shaped pits and clusters exist. New pits might be formed by desorption of dihydride and trihydride from the surface followed by $\mathrm{H}$-desorption around these defects.

Figure 9c shows the topography of the $\mathrm{H}-\mathrm{Si}(111)$ surface after annealing at $450{ }^{\circ} \mathrm{C}$ for $2 \mathrm{~h}$. The shapes of the steps and the terraces were preserved (not shown here) but the atomic arrangement changed remarkably and it seems that there is no long-range order as on the H-Si(111)- $(1 \times 1)$ surface. The clusters with a $1 \times 1$ structure disappeared, from which one can conclude that the clusters were neither contaminations (hydrocarbonates, carbides or metals) nor clusters of silicon oxide, which can normally be desorbed from the surface at temperatures significantly higher than $450{ }^{\circ} \mathrm{C}$. Thus they consist of silicon atoms, which is consistent with the results of Komeda et al. ${ }^{[36]}$ The structure becomes irregular and the surface looks very rough, but the root mean square (rms) roughness was almost the same as for the previously annealed surface shown in Figures $9 \mathrm{a}$ and $\mathrm{b}$. Several fragments of the DAS structures can be found on the surface after annealing at $550{ }^{\circ} \mathrm{C}$ but the density of these structures is far lower than in the case of 1 ML hotdeposited $\mathrm{Ag}$ at $550{ }^{\circ} \mathrm{C}$ (see Figs. 6 and 7). This experiment shows that the hot deposition of Ag plays an important role in the energy of formation of $\operatorname{Si}(111)-((2 n+1) \times(2 n+1))$ reconstructions.

After $1 \mathrm{~h}$ of additional annealing at $650^{\circ} \mathrm{C}$ the surface was converted into an almost perfect $7 \times 7$ reconstruction (see Fig. 9d). The transformation temperature (annealing temperature) is higher than in the case of hot deposition of Ag. One can observe relatively tall clusters (maximum height $3.0 \mathrm{~nm}$ ), which are thought to consist of silicon atoms, on top of a welldefined $7 \times 7$ reconstructed surface. These clusters are spread uniformly over the $\mathrm{Si}(111)-(7 \times 7)$ surface. Although there exist some defects in the $7 \times 7$ structure, the images show the same structure as obtained by known methods. ${ }^{[39-41]}$ The presence of silicon clusters might explain why no clear $7 \times 7$ diffraction patterns of this sample have yet been resolved using LEED and RHEED techniques.

\section{Conclusion}

To summarize, Co growth on H-passivated $\mathrm{Si}(111)$ was found to occur by the Vollmer-Weber (3D) growth mode at RT, leading to a surface with a peak-to-peak roughness of $18 \AA$ for a $200 \AA$ thick Co film. In the case of an increased growth temperature of $T=100{ }^{\circ} \mathrm{C}$, cobalt silicide is formed. The deposition of a Ag buffer layer does not reduce the peak-to-peak roughness or increase the homogeneity of a following Co layer. The performance of TMR and GMR components and devices grown on H-passivated $\mathrm{Si}(111)$ substrates in UHV should be investigated in the future.

Various DAS superstructures, such as $3 \times 3,5 \times 5,7 \times 7$, and $9 \times 9$, were observed on the $\mathrm{Si}(111)$ surface after the deposition of $1 \mathrm{ML} \mathrm{Ag}$ at a substrate temperature of $550^{\circ} \mathrm{C}$. All the metastable structures convert into the $7 \times 7$ reconstruction after additional annealing at $600{ }^{\circ} \mathrm{C}$. After $\mathrm{Ag}$ deposition at a substrate temperature of $T=250{ }^{\circ} \mathrm{C}$ followed by annealing at $550{ }^{\circ} \mathrm{C}$ no metastable DAS structures were found on the surface. Hydrogen thermal desorption experiments also did not show extensive formation of DAS structures at temperatures near $550^{\circ} \mathrm{C}$.

\section{Experimental}

Thin film deposition by molecular beam epitaxy (MBE) and in situ characterization of the samples were carried out using two different UHV systems. The first was an Omicron Surface Science UHV system with a base pressure of $8 \times$ $10^{-11}$ mbar equipped with an in situ atomic force microscope and a scanning tunneling microscope as well as electron beam evaporators. The second system was an UHV MBE system with a base pressure of $8 \times 10^{-11}$ mbar. It was equipped with electron beam evaporators and Knudsen cells for thin film deposition, and in addition in situ STM [42,43], AES, LEED, RHEED, and X-ray photoelectron spectroscopy (XPS) experiments were possible.

The substrates were rectangular $(10 \mathrm{~mm} \times 4 \mathrm{~mm} \times 0.375 \mathrm{~mm})$ cut from a B-doped p-type $\mathrm{Si}(111)$ wafer $(4.5 \Omega \mathrm{cm})$ within an out-of-plane misorientation angle of $\pm 0.5^{\circ}$ miscut. The sample cleaning and hydrogen $(\mathrm{H})$ termination were carried out in accordance with the wet-chemical etching procedure described by Higashi et al. [44]. This preparation procedure is known to yield an ideal monohydride-terminated $\mathrm{Si}(111)$ surface [12]. After the preparation process the samples were transferred to a load-lock chamber and pumped down by an oilfree prepump and a turbomolecular pump within $30 \mathrm{~min}$. The samples were introduced into the UHV chamber after the pressure in the load-lock reached $1 \times 10^{-7}$ mbar.

The H-Si(111) samples were heated using a radiative heater placed behind the sample holder while the pressure was kept below $5 \times 10^{-9}$ mbar. The temperature was monitored by a chromel-alumel thermocouple located between the heater and the sample holder. The absolute accuracy of the temperature measurements was $\pm 20^{\circ} \mathrm{C}$. After each heating process the samples were slowly cooled down to room temperature (cooling speed less than $0.5^{\circ} \mathrm{C} / \mathrm{s}$ ).

All STM measurements were carried out at room temperature using electrochemically etched polycrystalline tungsten tips cleaned in $\mathrm{UHV}$ by $\mathrm{Ar}^{+}$ion sputtering.

Received: November 24, 2000 Final version: February 5, 2001

[1] M. N. Baibich, J. M. Broto, A. Fert, F. Nguyen van Dau, F. Petroff, P. Etienne, G. Creuzet, A. Friederich, J. Chazelas, Phys. Rev. Lett. 1988, 61, 2427.

[2] G. Binasch, P. Grünberg, F. Saurenbach, W. Zinn, Phys. Rev. B 1989, 39, 4828.

[3] S. S. P. Parkin, N. More, K. P. Roche, Phys. Rev. Lett. 1990, 64, 2304.

[4] J. S. Moodera, L. R. Kinder, T. M. Wong, R. Meservey, Phys. Rev. Lett. 1995, 74, 3273

[5] S. S. P. Parkin, K. P. Roche, M. G. Samant, P. M. Rice, R. B. Beyers, R. E. Scheuerlein, E. J. O'Sullivan, S. L. Brown, J. Bucchigano, D. W. Abraham, Y. Lu, M. Rooks, P. L. Trouilloud, R. A. Wanner, W. J. Gallagher, J. Appl. Phys. 1999, 85, 5828

[6] M. Julliére, Phys. Lett. A 1975, 54, 225

[7] P. Zahn, J. Binder, I. Mertig, R. Zeller, P. H. Dederichs, Phys. Rev. Lett. 1998, 80,4309

[8] C. Blaas, P. Weinberger, L. Szunyogh, P. M. Levy, C. B. Sommers, Phys. Rev. B 1999, 60, 492

[9] J. Kudrnovsky, V. Drchal, C. Blaas, I. Turek, P. Weinberger, Phys. Rev. Lett. 1996, 76,3834

[10] I. I. Oleinik, E. Y. Tsymbal, D. G. Pettifor, Phys. Rev. B 2000, 62, 3952

[11] P. Mavropoulos, N. Papanikolaou, P. H. Dederichs, Phys. Rev. Lett. 2000 $85,1088$.

[12] P. Dumas, Y. J. Chabal, G. S. Higashi, Phys. Rev. Lett. 1990, 65, 1124.

[13] J. Camarero, L. Spendeler, G. Schmidt, K. Heinz, J. J. de Miguel, R. Miranda, Phys. Rev. Lett. 1994, 73, 2448.

[14] W. F. Egelhoff, M. T. Kief, Phys. Rev. B 1992, 45, 7795.

[15] W. J. Gallagher, S. S. P. Parkin, Y. Lu, X. P. Bian, A. Marley, K. P. Roche, R. A. Altmann, S. A. Rishton, C. Jahnes, T. M. Shaw, G. Xiao, J. Appl. Phys. 1997, 81, 3741 .

[16] T. Zhu, R. Swanson, presented at 43rd Conf. on Magnetism and Magnetic Materials, Miami, FL 1998.

[17] K. Kaji, S.-L. Yau, K. Itaya, J. Appl. Phys. 1995, 78, 5727.

[18] F. Nouvertné, U. May, M. Bamming, A. Rampe, U. Korte, G. Güntherodt, R. Pentcheva, M. Scheffler, Phys. Rev. B 1999, 60, 14382. 
[19] M. Scheffler, C. Stampfl, in Handbook of Surface Science, Vol. 2 (Eds: K. Horn, M. Scheffler), Elsevier, Amsterdam 1999.

[20] J. Derrien, M. De Crescenzi, E. Chainet, C. d'Anterroches, Phys. Rev. B 1987, 36, 6681.

[21] M. Naitoh, A. Watanabe, S. Nishigaki, Surf. Sci. 1996, 357-358, 140.

[22] G. van Tendeloo, X. B. Zhang, A. L. Vasiliev, Y. He, L.-M. Yu, P. A. Thiry, Surf. Sci. 1995, 340, 317.

[23] Binary Alloy Phase Diagrams, Vol.1 (Ed: T. B. Massalski), ASM International, Materials Park, OH 1996, p. 93.

[24] Binary Alloy Phase Diagrams, Vol. 1 (Ed: T. B. Massalski), ASM International, Materials Park, OH 1996, p. 25.

[25] K. Takayanagi, Y. Tanishiro, M. Takahasi, S. Takahashi, J. Vac. Sci. Technol. A 1985, 3, 1502

[26] K. J. Wan, X. F. Lin, J. Nogami, Phys. Rev. B 1992, 46, 13635

[27] H. Ohnishi, I. Katayama, Y. Ohba, F. Shoji, K. Oura, Jpn. J. Appl. Phys. 1993, 32, 2920.

[28] G. LeLay, Surf. Sci. 1983, 132, 169

[29] A. A. Saranin, A. V. Zotov, V. G. Lifshits, J. T. Ryu, O. Kubo, H. Tani, T. Harada, M. Katayama, K. Oura, Surf. Sci. 1999, 429, 127

[30] A. Shibata, Y. Kimura, K. Takayanagi, J. Vac. Sci. Technol. B 1994, 12, 2026.
[31] W. McComb, D. J. Moffatt, P. A. Hackett, B. R. Williams, B. F. Mason, Phys. Rev. B 1994, 49, 17139

[32] A. Shibata, Y. Kimura, K. Takayanagi, Surf. Sci. 1994, 303, 161.

[33] Y. N. Yang, E. D. Williams, Phys. Rev. Lett. 1994, 72, 1862.

[34] G.-X. Qian, D. J. Chadi, Phys. Rev. B 1987, 35, 1288.

[35] G.-X. Qian, D. J. Chadi, J. Vac. Sci. Technol. B 1986, 4, 1079.

[36] T. Komeda, Y. Morita, H. Tokumoto, Surf. Sci. 1996, 348, 153.

[37] Le Tanh Vinh, M. Eddrief, C. A. Sebenne, P. Dumas, A. Taleb-Ibrahimi, R. Gunther, Y. J. Chabal, J. Derrien, Appl. Phys. Lett. 1994, 64, 3308.

[38] In this case we deposited nominally $3 \mathrm{ML}$ of Ag, so only some of the deposited $\mathrm{Ag}$ took part in the formation of the $\mathrm{Ag}-\mathrm{Si}(111)-(\sqrt{3} \times \sqrt{3})$ phase and the remaining $\mathrm{Ag}$ would be clustered on the $\mathrm{Ag}-\mathrm{Si}(111)-(\sqrt{3} \times \sqrt{3})$ surface.

[39] K. Takahashi, C. Nara, T. Yamagishi, T. Onzawa, Appl. Surf. Sci. 1999, 151, 299.

[40] P. A. Bennet, M. B. Webb, Surf. Sci. 1981, 104, 74.

[41] S. Kitamura, T. Sato, M. Iwatsuki, Nature 1991, 351, 215.

[42] K. Besocke, Surf. Sci. 1987, 181, 145.

[43] J. Frohn, J. F. Wolf, K. Besocke, M. Teske, Rev. Sci. Instrum. 1989, 60, 1200.

[44] G. S. Higashi, Y. J. Chabal, G. W. Trucks, K. Raghavarachi, Appl. Phys. Lett. 1990, 56, 656 . 